\title{
Clinical Application of Pet
}

\author{
Francisco Lomeña $^{1 *}$ and Marina Soler ${ }^{2}$ \\ 1Nuclear Medicine Hospital Clínic Villarroel, 170; 08036; flomena@clinic.ub.es; Barcelona - Spain. 2PET Unit. \\ CETIR Grup Medic. Esplugues de Llobregat; Barcelona - Spain
}

\begin{abstract}
Positron emission tomography (PET) is an imaging modality that gives information on tissue metabolism and functionalism, different from other imaging techniques like computed tomography $(C T)$ and magnetic resonance imaging (MRI), which provide anatomical or structural information. PET has reached its development in biomedical research because of its capacity to use analogous compounds of many endogenous substance as tracers, and to measure, in vivo and in a non-invasive way, their consumption by the different organs and tissues of the mammalian body. Fluordeoxyglucose-F18 (FDG) PET has been proven to be a tracer adequate for clinical use in oncology and in many neurological diseases, with an excellent cost-efficiency ratio. The current PET-CT scanners can come to be the best tools for exploring patients who suffer from cancer.
\end{abstract}

Key words: PET, fluordeoxyglucose-F18 (FDG), cancer, PET/CT

\section{INTRODUCTION}

The morphologic resolution achieved with the current PET scanners is very good, $6-8 \mathrm{~mm}$ in most of the commercial models. Its sensitivity to detect a regional increase or decrease in a tracer's concentration is high, detecting changes at a nanomolar range. Hybrid scanners PET/CT allow the acquisition of PET and CT images at the same time and in a single study, visualizing them separately or fused. Therefore, CT anatomical information and PET functional or metabolic information are contained in the same images (Phelps M, 2000).

Many substances, including endogenous molecules, can become PET tracers for visualization and measurement of many intracellular biochemical processes. The positron-emitting radio nuclides most used are cyclotron products, like $\mathrm{C}-11, \mathrm{~N}-13, \mathrm{O}-15$ and F-18. They permit an easily labelling, replacing some atoms of $\mathrm{O}, \mathrm{C}, \mathrm{N}$ or $\mathrm{H}$ by $\mathrm{O}-15, \mathrm{C}-11$ or $\mathrm{N}-13$ or F-18, respectively, in the ligands. They have a very short half-life (O-15, 2 minutes; $\mathrm{N}$ 13, 10 minutes; C-11, 20 minutes; F-18, 110 minutes). Because of this short half-life, the cyclotron must be located in its own nuclear medicine department, except for some F-18 derivatives, like FDG, that can be produced by an external radiopharmacy unit with a cyclotron, and after delivered to different PET facilities.

\section{CLINICAL APPLICATIONS OF PET}

Currently, FDG is the unique PET tracer with clinical applications. FDG is a glucose analogous that penetrates into the cells using sodium-glucose transporters and specific glucose membrane transporters. Once inside the cell, FDG is phosphorylated by the action of

\footnotetext{
Author for correspondence
} 
hexokinase to FDG-6-phosphate (FDG-6-P) and remains trapped in tissue, whereas glucose is not. From there, FDG-6-P does not follow the metabolic route of glycolysis or glycogen synthesis, being retained in the cell. The physiological or normal distribution of FDG in the organism corresponds to the cellular and tissue consumption of endogenous glucose. Brain is the organ that shows the major glucose uptake. FDG uptake in heart and skeletal muscles is variable and depends on glucose blood levels (4 - 6 hours fasting, previous to FDG injection, is mandatory), endogenous insulin concentration, and muscular contraction. Bowel uptake is also variable, depending on the peristaltism and muscular tone. There is urinary elimination of FDG; kidneys, ureteral tracts and urinary bladder are visualized in PET images.

PET in cardiovascular diseases: Different authors have demonstrated FDG uptake in severely hypoperfused myocardial regions. The flow-metabolism dissociation pattern indicates the presence of ischemic but viable myocardium. At the moment, FDG uptake measured by PET is considered the gold standard to diagnose myocardial viability. The main application of FDG-PET in cardiovascular pathology is to determine the myocardial viability in patients who will be submitted to coronary surgery. If there is no myocardial viability by PET, patients will be treated medically or included in a transplant waiting list (Schwaiger M and Hicks R, 1991).

PET in Central Nervous System (CNS) diseases: It is accepted that FDG-PET is the most exact in vivo imaging methodology to evaluate global and regional cerebral metabolism. In the USA, in September, 2004, the HCFA and Medicare established that FDGPET can be used in those patients with moderate cognitive impairment to make the differential diagnosis between Alzheimer dementia and front-temporal dementia. The evaluation of patients with partial epilepsy, refractory to medical treatments, before the surgery is also recognized as an appropriate use of FDG-PET,. FDG-PET detects, during the interictal phase, the epileptogenous focus as a hypometabolic focal area in the cerebral cortex (Newberg A, 2002).
PET in cancer: The main applications of FDG PET are related to the diagnosis, staging, therapy monitoring, and prognosis of patients with cancer. PET findings have a direct clinical impact on the patient management. FDG-PET can show the pathological increase in glucose consumption that the tumour cells present in vitro. Phosphatase absence in the tumour cells causes an intense metabolic retention of FDG-6P. A high contrast between the tumour cell uptake and the healthy cell uptake results in a high detection sensitivity by PET. FDG uptake is related to the high cellularity and the cellular proliferation and, therefore, to the degree of malignancy. The most aggressive tumours require greater glucose consumption to maintain their accelerated growth, whereas those of low degree have less FDG uptake. Scars and already established necrosis and oedema do not show FDG uptake.

PET is a good diagnostic tool in oncology because: first, one can do a whole body study in the same exploratory act; second, it has a great sensitivity for detecting malignant neoplastic tissue, demonstrating tumour infiltration in normal sized lymph nodes and in organs that do not yet present anatomical alterations in CT or MRI; third, the findings are less artefacted by the therapy than those of CT and MRI; thus, PET can distinguish residual post-therapy fibrosis and/or postsurgical anatomical distortion from viable tumour tissue; finally, it has a relatively high negative predictive value, since a normal study almost totally discards malignancy - macroscopic tumour disease.

FDG-PET can have applications in several clinical situations: to support a diagnosis of a benign lesion or malignancy in processes detected by other techniques, but with complicated or impossible histological confirmation; to establish the extension of an already known tumour prior to treatment staging; to differentiate neoplastic tissue from fibrotic residual masses after surgery, chemotherapy and/or radiotherapy; to locate a tumour recurrency suspected by clinical analysis and/or an increase in tumour markers; to do a new study of extension after recurrence diagnosis - re-staging; to evaluate the early therapeutic effects; and to look for the primary tumour in a patient with metastatic disease of unknown origin or para-neoplastic syndrome. In addition, PET may guide needle aspiration or 
biopsy and allows the definition of the volume of irradiation in radiotherapy planning.

Few false negatives have been described. Hyperglycaemia can be a cause for them, due to the competition between FGD and the endogenous glucose. In order to avoid them, FDG must be administered to the patient when glycaemia is lower than $140 \mathrm{mg} / \mathrm{dL}$. It is necessary to remember that FDG uptake can be low in some necrotic, mucinous tumours and in low grade tumours. Tumours of small size - and micrometastases - can also occur unnoticed. It is very difficult to speak about diameter or size thresholds for detection, since PET measures the uptake or contrast ratio between lesion and tissue. This ratio is usually very high in the malignant processes.

Another disadvantage of FDG-PET is the possibility of false positive results for nonmalignant diseases like inflammations, infections and granulomas (sarcoidosis, tuberculosis, histoplasmosis, aspergyllosis, etc) that can present FDG uptake similar to the malignant tumors (Cabrera A, 2002; Cabrera A, 2002; Gamez C, 2002).

FDG-PET in Oncology: In this section, PET applications in different tumors is described.

Brain tumors: FDG-PET allows the differential diagnosis between high-grade tumor-like relapse and radionecrosis, being more specific than $\mathrm{CT}$ and MRI. MRI is superior to FDG-PET in the detection of cerebral metastases, since these, when they are asymptomatic, present a small size and usually have a cortical-subcortical location, being unnoticed by FDG-PET.

Head and neck tumors: CT and MRI are the most appropriate techniques to determine local invasion of a primitive head and neck tumor, but FDG-PET is more sensible and specific in the detection of lymph node and metastatic disease.

Lung Cancer: The established FDG-PET indication is the differential diagnosis of solitary pulmonary nodules when they are undetermined by CT. False positives have been described for some granulomas, like those of tuberculosis. A positive PET forces one to discard cancer. NonFDG-uptake in a radiological nodule of diameter $>1 \mathrm{~cm}$ suggests benignancy with a high negative predictive value for cancer, which allows one to adopt an expectant attitude. Another indication is the study of mediastinal staging of non-small cell lung cancer (NSCLC).
For several authors, a negative mediastinal PET supposes a N0 stage, whereas a positive PET forces one to execute mediastinoscopy or surgery to corroborate nodal infiltration. In addition, FDG-PET can discover metastatic disease in a presurgical diagnosis extension in $10-20 \%$ of NSCLC, contra-indicating radical surgery. PET changes the therapeutic approaches of NSCLC patients in $40 \%$ of the cases, detecting a greater extension of the disease in many of those classified initially as surgical. PET also may play a role in the suspicion of recurrence and in the re-staging.

Digestive cancers: FDG-PET is indicated in esophagus presurgical staging. Another established indication is for the clinical, analytical and/or radiological suspicion of colorectal cancer recurrence to determine its location and to perform the re-staging, with the aim of evaluating the possibility of radical surgery. It is also useful in those patients with colorectal cancer already operated who develop liver metastases, because a new surgery must be contraindicated if extra-hepatic disease is detected by PET.

Lymphomas: FDG-PET detects more lesions than CT in the initial staging and the re-staging of non-Hodgkin's and Hodgkin's disease, increasing the stage in $8-16 \%$ of the cases. PET is used for valuation of therapy response, differentiating with better accuracy if a residual post-therapy mass is scar or corresponds to tumor persistence. In addition, FDG-PET posttherapy uptake has an added prognosis value, with greater index of recurrences in patients with positive PET. PET is also useful in evaluating patients if there exists the possibility of relapse, when clinical, analytical, radiological and scintigraphic (67Ga) examinations are not in agreement. PET images have better morphologic resolution than Ga67 SPET, and greater sensitivity, mainly in low-grade lymphomas.

Melanomas: FDG-PET is indicated in the initial staging study of melanomas with high probability of metastatic disease or in the case of recurrence.

Breast Cancer: It is currently accepted that FDG-PET is the election method for detecting and to do staging in recurrent and/or metastatic disease to establish the therapeutic strategy. FDG-PET seems more accurate and sensible for detecting small bone metastasis than bone scintigraphy, especially when they are located in 
the medullar space. FDG-PET is used to evaluate chemotherapy response in cases of locally advanced primitive tumours, prior to surgery, and also in non-surgical multisystemic relapses.

Thyroidal Cancer: FDG-PET is indicated for patients with thyroidectomy for differentiated thyroid cancer that, in their evolution, present thyroglobulin level elevation with negative 131I scan. It is also useful for detecting residual or recurrent disease of already operated medullary carcinomas that present calcitonin level elevation.

Genitourinary tract tumor: The normal urinary FDG elimination makes the interpretation of these types of tumors difficult. In addition, the special histology of the urinary tumours does not cause elevated FDG uptake. This is the case for prostate cancer. It has been described that other tracers, like coline-C11 or acetate-C11, present better sensitivity and specificity in patients with recurrent or metastatic prostate gland cancer.

Unknown origin cancer: FDG-PET has been able to locate the primitive tumor in $24-53 \%$ of the patients with metastatic disease from an unknown origin tumour. Its clinical impact is important in patients with removable or radically curable disease, as happens in cases with cervical or supraclavicular lymph nodes, in which a head and neck tumour is finally detected. Also, it seems useful in patients with multiple metastatic diseases in which the location of the primitive tumour allows a more specific chemotherapy. Another indication described for FDG-PET is the search for the primitive tumour in patients with neurological para-neoplastic syndromes, in which the presence of anti-neuronal antibodies has been demonstrated. In these cases, the fact of being able to locate and treat the tumour can stabilize or minimize the neurological symptoms (Hustinx R, 2002; Lomeña F and Simo M, 2003).

\section{CLINICAL APPLICATIONS OF PET/CT}

At the moment, CT is the essential tool in clinical management of patients with cancer for the initial diagnosis - possibility for guiding the biopsy - for the diagnosis of loco-regional staging, in the control of therapy response, in the clinical follow-up and for the detection of recurrences. The best characteristic of CT is its extraordinary morphologic resolution to define exactly the anatomical location of the tumour and to delimit his local extension. CT uses morphometric criteria to detect disease, evaluating the size and the density of the anatomical structures. The tumours appear as a size increase or as a distortion of anatomical structures. For example, a lymphatic node can be infiltrated by a tumour when its smaller diameter is greater than $1 \mathrm{~cm}$. The tumour can be represented like an alteration of the density, being more or less dense that the healthy tissue that surrounds it. When there is a small difference between the abnormal density and the tissue that surrounds it, the use of endovenous contrast can increase the relation between the lesion and the normal tissue, increasing the sensitivity of CT detection. It is necessary to know that some inflammatory lesions and benign tumours present CT findings similar to the malignant tumours. Scars and changes resulting from the local treatment (surgery, radiotherapy) produce an anatomical distortion that makes the interpretation of CT findings difficult.

PET provides poor anatomical information. Tumours are appraised like isolated hypermetabolic areas, but one is not able to know their exact location, their anatomical extension or their limits. PET-CT images maintain the best characteristics of PET and CT and avoid their drawbacks. It is possible to obtain fused PET-CT images of the whole body (volumetric - in $3 \mathrm{D}$, axial tomography, coronal tomography) with a high ability of detect tumours, with high resolution of contrast and with high morphologic resolution, which allows one to define the anatomical extension of tumour infiltration and its limits.

The largest series of patients explored by PETCT hybrid scanners include those with head and neck tumors, NSCLC, and abdominal tumors (gynaecological, pancreas and colorectal cancers). In these patients, physiological FDG uptake in healthy anatomical structures (in neck: lymphatic structures, vocal cords, salivary glands, muscles, thyroid gland; in abdomen: intestinal uptake, urinary elimination) can be differenciated from malignant tumour uptake. In addition, the local and regional extension of the 
tumour can be defined (vascular invasion, pleural invasion, pericardium and thoracic wall infiltration by pulmonary cancers or the lymphatic node infiltrated by this type of tumour). PET-CT sensitivity is higher than the sensitivity of the PET alone and the CT alone, maintaining the same specificity. Some pending methodological questions are being implemented, such as the use of oral or endovenous contrasts during the CT acquisition, avoiding the artifacts generated in attenuationcorrected PET images.

The applications in which PET-CT can contribute more and could give better information are staging ("T" staging of the T1T4 tumours) prior to surgery or radiotherapy; detection, location of the infiltrated regional lymphatic nodes ("N" staging of the tumors: NON3); residual mass characterization after surgery, radiotherapy and/or chemotherapy; exact location of metastasis and recurrences, to perform a radical treatment; biopsy guide; definition of irradiation volumes in radiotherapy planning and to prepare the strategy for the surgical procedure to extirpate all or most of a tumour mass.

The use of hybrid PET/CTsystems will change the established PET clinical indications in patients with cancer. We are probably near a generic application. PET-CT can become an exploration of mandatory use in patients with cancer of any type, for staging at the moment of the initial diagnosis, to assess therapy effects and to evaluate the clinical suspicions of recurrence (Czernin J, 2004).

\section{RESUMO}

A tomografia por emissão de pósitrons (PET) é uma técnica de diagnóstico por imagem que fornece informação sobre o metabolismo e funcionamento dos tecidos, diferente de outras técnicas de imagens como tomografia computadorizada (TC) e ressonância magnética (RM), as quais fornecem informações estruturais ou anatômicas. O PET alcançou seu desenvolvimento em investigação biomédica devido à sua capacidade de usar traçadores análogos a muitas substâncias endógenas e de medir in vivo e de forma não invasiva seu consumo em diferentes órgãos e tecidos dos mamíferos 18Fluordesoxiglicose (FDG) PET tem provado ser uma exploração de uso clínico com excelente relação custo benefício em oncologia e em muitas doenças neurológicas. Os atuais tomógrafos por PET-CT podem chegar a ser a melhor ferramenta de diagnóstico nos pacientes que sofrem de câncer.

\section{REFERENCES}

Cabrera A, Gamez C, Martin JC. (2002), La tomografía por emisión de positrones (PET) en Oncología (Parte II). Rev Esp Med Nucl, 21, 131147.

Cabrera A, Gamez C, García-Belloso MJ. La tomografía por emisión de positrones (PET) en Oncología (Parte III). Rev Esp Med Nucl 2002; 21:304-320.

Czernin J. PET/CT: Imaging Function and Structure. J Nucl Med 2004; 45:1s-103s

Gamez C, Cabrera A, Sopena R, García-Belloso MJ. La tomografía por emisión de positrones (PET) en Oncología (Parte I). Rev Esp Med Nucl 2002; 21, 41-60.

Hustinx R, Benard F, Alavi A. Whole-Body FDGPET Imaging in the Management of patients with cancer. Seminars in Nuclear Medicine 2002; vol XXXII, num. 1; p.35-46.

Lomeña $\mathrm{F}$ and Simo M. Indications of PET imaging. Med Clin (Barc) 2003; 120:742-749.

Newberg A, Alavi A, Reivich M. Determination of regional cerebral function with FDG. Seminars in Nuclear Medicine 2002; vol XXXII, num. 1; p.1334.

Phelps M. Positron Emission Tomography provides molecular imaging of biological processes. PNAS 2000; 1, 9226-9233.

Schwaiger M, Hicks R. The clinical role of metabolic imaging of the heart by positron emission tomography. J Nucl Med 1991; 32, 565-578. 